\title{
Antireflux valve metal stent versus conventional self-expandable metal stent in distal malignant biliary obstruction: a systematic review and meta-analysis
}

\author{
Anas Renno , Yousef Abdel-Aziza, Tamer Ahmed ${ }^{a}$, Yaseen Alastal ${ }^{a, b}$, Javaid Toseef ${ }^{a, b}$, Yasir Al-Abboodia, \\ Ali Nawras ${ }^{\mathrm{a}, \mathrm{b}}$ \\ University of Toledo Medical Center, Toledo, OH, USA
}

\begin{abstract}
Background In patients with distal malignant biliary obstruction (MBO), endoscopic biliary drainage using the conventional self-expandable metal stent (SEMS) is the gold standard method for palliative treatment. However, there are limited data on the role of the antireflux valve metal stent (ARVMS). The aim of this study was to compare the safety and efficacy of ARVMS and SEMS in patients with distal MBO.
\end{abstract}

Methods We searched PubMed, Ovid, Embase and the Cochrane Library from inception until April 2019 for relevant randomized controlled trials (RCTs). The selected studies provided data regarding technical and clinical success rates, adverse events, and stent dysfunction. Data were meta-analyzed using RevMan software.

Results Three RCTs were selected, enrolling 293 patients (147 ARVMS and 146 SEMS). The rates of technical success were $95.23 \%$ and $99.31 \%$ for ARVMS and SEMS groups, respectively (odds ratio $[\mathrm{OR}]$ 0.13, 95\% confidence interval [CI] 0.01-1.06; $\mathrm{P}=0.06$ ). The clinical success rates were $91.57 \%$ and $89.36 \%$ for ARVMS and SEMS groups, respectively (OR 1.30, 95\%CI 0.48-3.51; $\mathrm{P}=0.61$ ). There was no significant difference between the ARVMS and SEMS groups in terms of adverse events (OR 0.61, 95\%CI 0.35-1.05; P=0.07) or stent dysfunction (OR 0.77, 95\%CI 0.31$1.95 ; \mathrm{P}=0.58$ ), while the incidence of stent occlusion was significantly lower in the ARVMS group (OR 0.44, 95\%CI 0.26-0.76; $\mathrm{P}=0.003$ ).

Conclusion Our study showed that ARVMS and SEMS had similar technical and clinical success rates. Adverse events were comparable between the 2 arms; however, ARVMS was associated with a lower risk of stent occlusion. Larger RCTs are required to verify the benefit of ARVMS in distal $\mathrm{MBO}$ patients.

Keywords Biliary malignancy, biliary obstruction, metal stent, meta-analysis

Ann Gastroenterol 2019; 32 (6): 1-9

${ }^{a}$ Department of Internal Medicine (Anas Renno, Yousef Abdel-Aziz, Tamer Ahmed, Yaseen Alastal, Javaid Toseef, Yasir Al-Abboodi, Ali Nawras); ${ }^{b}$ Division of Gastroenterology and Hepatology, University of Toledo Medical Center (Yaseen Alastal, Javaid Toseef, Yasir Al-Aboodi, Ali Nawras), OH, USA

Conflict of Interest: None

Correspondence to: Dr. Anas Renno, MD, University of Toledo Medical Center, Department of Internal Medicine, 3000 Arlington Avenue, Toledo, OH 43614, USA,

e-mail: anas.renno@utoledo.edu

Received 16 July 2019; accepted 23 September 2019; published online 21 October 2019

DOI: https://doi.org/10.20524/aog.2019.0427

\section{Introduction}

The conventional self-expandable metal stent (SEMS) has been the gold standard for palliative treatment of nonresectable distal malignant biliary obstruction (MBO) because of its longer duration of patency and better conformability to the bile duct compared with plastic stents [1]. However, cholangitis occurs in $6.5-22 \%$ of patients with SEMS [2-4]. This adverse event is mainly predicted by the transpapillary insertion of SEMS (which impairs sphincter function) [5] and probably occurs as a result of enteric biliary reflux (as reported in a previous study with barium examination) [6].

The antireflux valve metal stent (ARVMS) has been introduced as an alternative to SEMS to reduce the risk of 
cholangitis [7]. The addition of valves of different shapes to the stent was aimed to prevent biliary reflux. Some studies have compared the safety and efficacy of ARVMS to SEMS in patients with distal MBO [7-9]. However, there is still debate regarding the superiority of either of these 2 stent types. For example, Hu et al concluded that ARVMS significantly reduced the risk of ascending cholangitis and had longer stent patency [7]. On the other hand, Hamada et al showed that ARVMS did not increase the time to recurrent biliary obstruction and was associated with a higher rate of stent migration [8].

This analysis is an attempt to synthesize the evidence from published randomized controlled trials regarding the comparative safety and efficacy of AVRMS and SEMS stents in patients with distal MBO. To our knowledge, this is the first meta-analysis to be conducted with the intention of comparing both stents in this patient population.

\section{Materials and methods}

\section{Literature search}

This meta-analysis was conducted in accordance with the guidelines of the Preferred Reporting Items for Systematic Review and Meta-analyses (PRISMA) statement [10]. We searched PubMed, Medline (Ovid), Embase, and the Cochrane Library for randomized controlled trials (RCTs) published up to and including April 2019. We employed descriptors available from the Medical Subject Headings (MeSH) and, to a lesser degree, other related terms, aiming at a more sensitive strategy. The following terms were used to find eligible trials: antireflux metal stent OR self-expandable metal AND malignant biliary obstruction. Two reviewers (AR,YA) independently screened the database search results for titles and abstracts. If either reviewer felt that a title and abstract met the eligibility criteria of our study, the full text of the study was retrieved.

\section{Study eligibility criteria}

Only published RCTs were considered eligible, without barriers as to the language or year of publication. We included RCTs that evaluated patients $>18$ years old, diagnosed with distal MBO (based on lab results and imaging), and undergoing primary drainage of the biliary tract via ARVMS or SEMS. Studies evaluating patients with benign biliary obstruction or published as abstracts only were excluded.

\section{Data extraction}

Data related to ARVMS and SEMS biliary drainage of MBO were collected using a preformatted Excel workbook. The data collected included technical and clinical success rates, as well as the duration of the procedure, adverse events, stent patency, and stent dysfunction. In our quantitative analysis, we used the absolute values, means, and standard deviations. If a study expressed outcomes using median and interquartile range, mathematical formulas were used for data conversion. In cases where the study failed to present means and standard deviations or median and interquartile ranges for the continuous variables of specific outcomes, the variable in question was excluded from the outcome analysis.

\section{Risk of bias assessment (quality assessment)}

We assessed the risk of bias in each trial using the Cochrane risk of bias tool for RCTs. Reviewers provided an evaluation for risk of bias as low (bias is not present or unlikely to alter the results seriously), unclear, or high (bias may seriously alter the results) for each of the following domains: sequence generation, allocation sequence concealment, blinding of participants and surgical staff, blinding of outcome assessment, selective outcome data, incomplete outcome data, and other biases [11]. Reviewers' disagreements on risk of bias judgments were resolved by an independent third reviewer.

\section{Publication bias}

A funnel plot was used to assess for publication bias.

\section{Data analysis}

For dichotomous variables, we calculated the odds ratios(ORs), using the Mantel-Haenszel test, together with the corresponding 95\% confidence intervals (CI). The results were displayed as forest plots. We assessed the heterogeneity among studies using the Cochrane $\mathrm{Q}$ and $I$-square $\left(I^{2}\right)$ tests, based on which the fixed- or random-effects model was used (for homogenous and heterogeneous datasets, respectively) [12]. All analyses were carried out using Review Manager software (RevMan 5.3.5; Cochrane Collaboration, Oxford, United Kingdom).

\section{Results}

\section{Literature search results}

The electronic search of four databases retrieved 254 studies. This number was reduced to 6 after title/abstract screening: i.e., 6 full-text articles were retrieved for full-text screening. Three studies were excluded for not meeting our eligibility criteria and 3 studies were selected for qualitative and quantitative analysis. The search and study selection strategies are illustrated in Fig. 1.

\section{Characteristics of included studies}

The 3 eligible studies enrolled a total sample size of 293 patients (147 ARVMS and 146 SEMS). Only one trial 


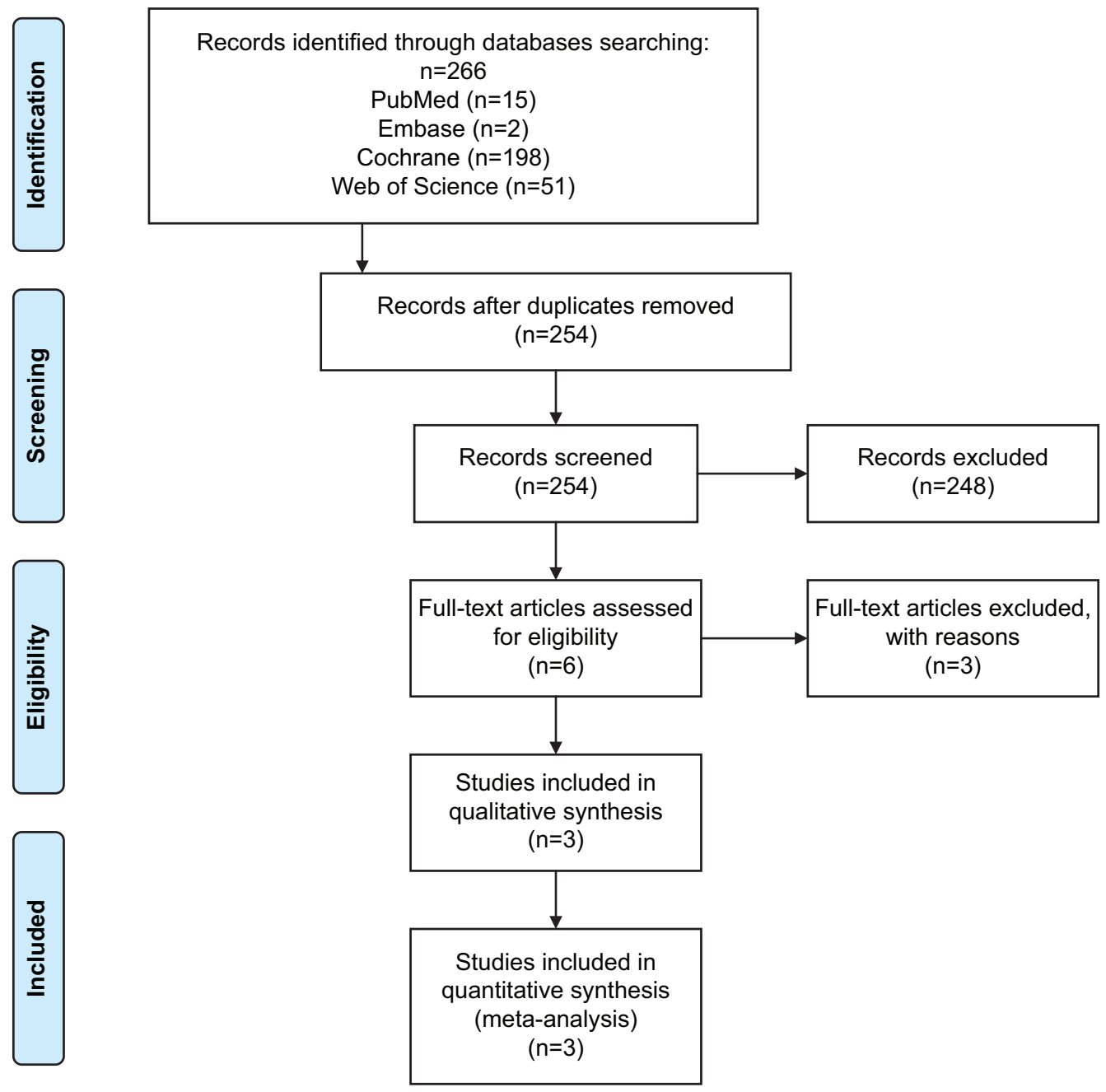

Figure 1 PRISMA flow diagram of literature search and study selection

was multicenter; the other 2 were single-center studies. All studies enrolled patients with distal unresectable malignant obstruction in the biliary tract. All 3 studies were conducted in East Asia (Japan, China, and South Korea). The patients' mean age ranged between 66.3 and 74.5 years, with males representing $54.9 \%$ of the pooled population. The characteristics of the eligible studies and their enrolled patients are summarized in Table 1.

\section{Risk of bias assessment results}

All 3 studies had a low risk of bias in terms of random sequence generation, allocation concealment, incomplete outcome data, and selective data reporting. The studies were mostly open-label; they had high risks of bias in terms of participants and blinding of outcome assessors (except for 1 study). Insufficient data were provided to assess the existence of other sources of bias. The risk of bias assessment results for the included studies are summarized in Fig. 2.

\section{Efficacy outcomes}

The rates of technical success were $95.23 \%$ and $99.31 \%$ for ARVMS and SEMS groups, respectively. The clinical success rates were $91.57 \%$ and $89.36 \%$, respectively. Pairwise meta-analysis showed no significant difference between the ARVMS and SEMS groups in terms of technical success (OR 0.13, 95\%CI 0.01-1.06; $\mathrm{P}=0.06$; Fig. 3A) or clinical success ( $\mathrm{OR} 1.30$, 95\%CI 0.48-3.51; $\mathrm{P}=0.61$; Fig. 3B). No significant heterogeneity was noted $\left(\mathrm{P}=0.86, I^{2}=0 \%\right)$; therefore, the analysis was conducted using the fixed-effects model.

\section{Safety outcomes}

The most common adverse event was cholangitis, occurring in $8.6 \%$ and $17.2 \%$ of the AVRMS and SEMS groups, respectively. This was followed by pancreatitis, occurring in $5.7 \%$ and $8.9 \%$, respectively. Other less frequent 


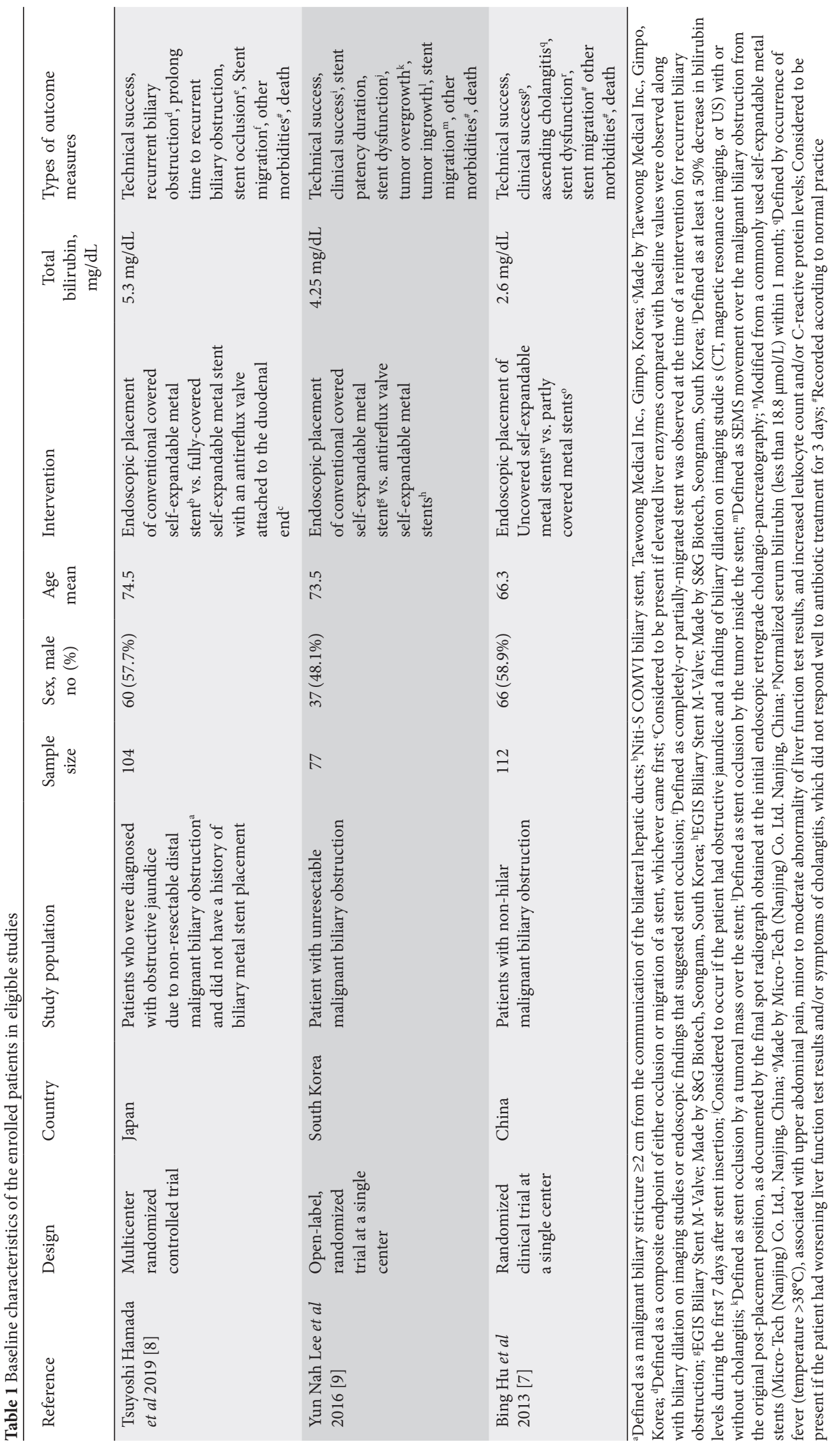


adverse events included cholecystitis, bleeding, liver abscess, and abscess around the bile duct (Table 2). Pairwise meta-analysis showed no significant differences between ARVMS and SEMS groups in terms of the frequency of all (OR 0.61, 95\%CI 0.35-1.05; P=0.07; Fig. 4A), early (OR 0.69, 95\%CI 0.27-1.81; $\mathrm{P}=0.45$; Fig. $4 \mathrm{~B}$ ) and late (OR $0.47,95 \% \mathrm{CI}$ 0.22-1.02; $\mathrm{P}=0.05$; Fig. $4 \mathrm{C}$ ) adverse events. No significant heterogeneity was noted across the 3 analyzed outcomes $(\mathrm{P}>0.1)$. The funnel plot was symmetrical, consistent with a lower likelihood of publication bias (Fig. 5).

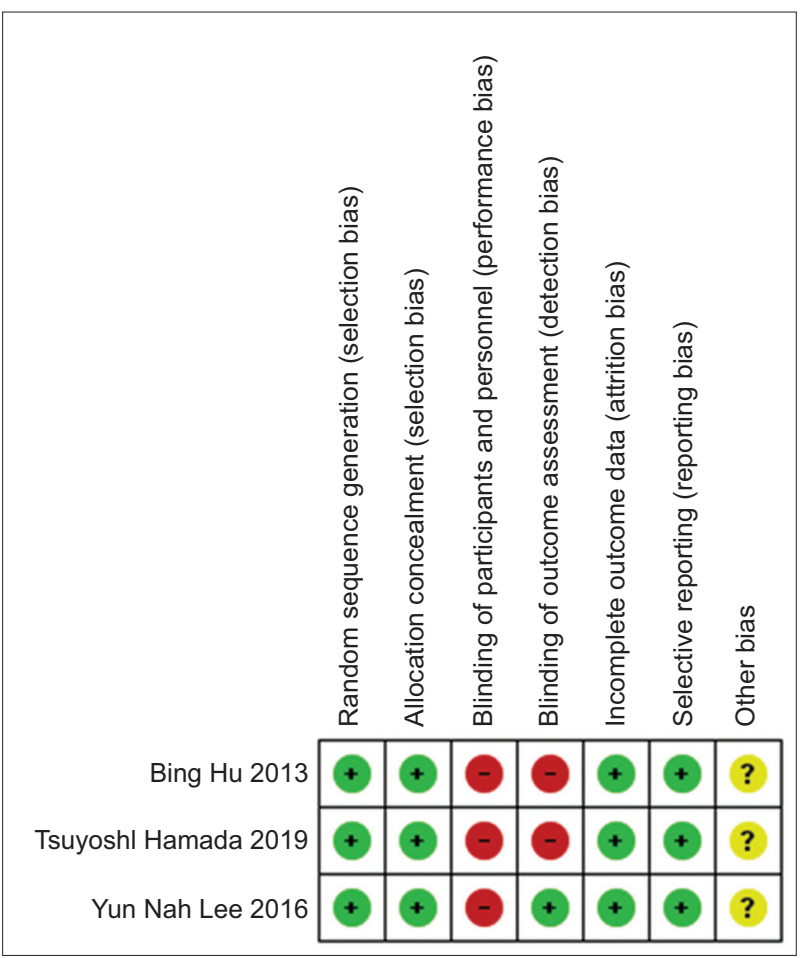

Figure 2 Risk of bias assessment results according to the Cochrane risk of bias tool

\section{Stent dysfunction}

The most common cause of stent dysfunction was stent migration, recorded in $16.2 \%$ and $7 \%$ of the AVRMS and SEMS groups, respectively. This was followed by sludge occlusion, tumor ingrowth, and unknown causes (Table 3). Pairwise meta-analysis showed no significant difference between the stent types in terms of the overall incidence of stent dysfunction (OR 0.77, 95\%CI 0.31-1.95; $\mathrm{P}=0.58$; Fig. 6A). Significant heterogeneity was noted $\left(\mathrm{P}=0.03, I^{2}=72 \%\right)$ among the studies [7-9], best resolved when the study by Hamada et al [8] was excluded, while the new effect estimate showed a significant reduction in the overall incidence of stent dysfunction in favor of the AVRMS group. However, subgroup analysis by cause of dysfunction showed a greater frequency of stent migration (OR 2.76, 95\%CI 1.24-6.17; $\mathrm{P}=0.01$; Fig. 6B) and a lower frequency of stent occlusion (OR 0.44, 95\%CI 0.26-0.76; P=0.003; Fig. 6C) in the AVRMS group, in comparison to the SEMS group. The funnel plot was symmetrical, consistent with a lower likelihood of publication bias (Fig. 7).

\section{Discussion}

This meta-analysis showed that ARVMS exhibited comparable efficacy and safety profiles to SEMS, evident by the similarities in the rates of clinical and technical success, adverse events and stent dysfunction. No heterogeneity was detected across all outcomes, except in the outcome of overall adverse events. When this heterogeneity was resolved using the leave-one-out sensitivity analysis, a favorable safety profile appeared for ARVMS over SEMS. Interestingly, a lower rate of stent occlusion but a higher rate of stent migration were observed for ARVMS in comparison to SEMS.

ARVMSs were developed based on the notion that preventing biliary reflux using a valve may decrease stentassociated cholangitis [7]. Indeed, we observed from the pooled

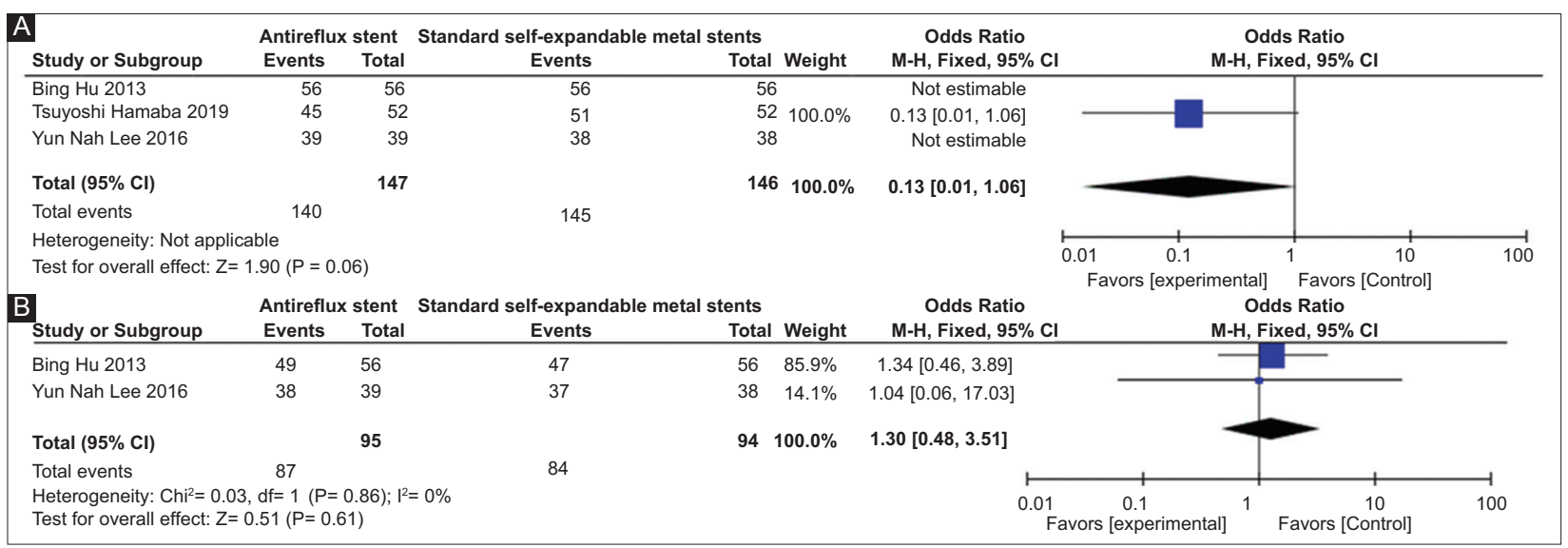

Figure 3 Forest plots representing odds ratios for (A) technical success, and (B) clinical success in the antireflux valve metal stent and the conventional self-expandable metal stent groups 
Table 2 Frequency of adverse events and stent dysfunction in groups that received antireflux valve and conventional self-expandable metal stents

\begin{tabular}{lcc}
\hline Adverse events & $\begin{array}{c}\text { Antireflux } \\
\text { stent } \\
(\mathrm{N}=140)\end{array}$ & $\begin{array}{c}\text { Self-expandable } \\
\text { metal stent } \\
(\mathrm{N}=145)\end{array}$ \\
\hline Pancreatitis & $8(5.7 \%)$ & $13(8.9 \%)$ \\
Cholangitis & $12(8.6 \%)$ & $25(17.2 \%)$ \\
\hline Cholecystitis & $5(3.6 \%)$ & $4(2.7 \%)$ \\
Bleeding & $2(1.4 \%)$ & $1(0.7 \%)$ \\
\hline Liver abscess & $2(1.4 \%)$ & $1(0.7 \%)$ \\
\hline Abscess around the bile duct & $1(0.7 \%)$ & $0(0 \%)$ \\
\hline Data are frequency (percentage) & & \\
\hline
\end{tabular}

Table 3 Frequency of stent dysfunction events in groups that received antireflux valve and conventional self-expandable metal stents

\begin{tabular}{|lcc}
\hline Stent dysfunction & $\begin{array}{c}\text { Antireflux } \\
\text { stent } \\
(\mathrm{N}=136)\end{array}$ & $\begin{array}{c}\text { Self- } \\
\text { expandable } \\
\text { metal stent } \\
(\mathrm{N}=144)\end{array}$ \\
\hline Tumor ingrowth & $5(3.7 \%)$ & $17(11.8 \%)$ \\
\hline Tumor overgrowth & $1(0.7 \%)$ & $9(6.2 \%)$ \\
\hline Sludge occlusion & $12(8.8 \%)$ & $14(9.7 \%)$ \\
\hline Food occlusion & $0(0 \%)$ & $1(0.7 \%)$ \\
\hline Stent migration & $22(16.2 \%)$ & $10(7 \%)$ \\
\hline Valve dysfunction & $2(1.5 \%)$ & $0(0 \%)$ \\
\hline Hemobilia & $1(0.7 \%)$ & $1(0.7 \%)$ \\
\hline Unknown causes & $7(5.1 \%)$ & $10(7 \%)$ \\
\hline
\end{tabular}

Data are frequency (percentage) data of the included studies that patients who received ARVMS developed markedly fewer cholangitis events than those who received SEMS. This finding is probably due to the antireflux valve. However, Hamada et al reported that the antireflux valve was not durable enough to prevent the duodenobiliary reflux after a long follow up [8]. However, one aspect that must be noted is the different shapes and lengths of valves used in the 3 available studies: nipple-shaped, funnel-shaped and windsockshaped.

Another interesting finding in our study was the lower risk of stent occlusion in ARVMS compared with SEMS. This may be because: (i) the large lumen of SEMS may predispose to reflux and accumulation of enteric contents; and (ii) uncovered stents, such as those used in the study by $\mathrm{Hu}$ et al, may suffer from tumor ingrowth through their mesh openings [13-15]. The initial event in stent occlusion appears to be the formation of a biofilm from bacterial and host proteins adhering to the stent wall. Bacterial enzymes then act on bile components, forming biliary sludge (bacterial products, calcium bilirubinate, and calcium fatty acid soaps) [16,17]. This finding was again debated in the study by Hamada et al who showed no prolongation of patency benefit for ARVMS over SEMS, but acknowledged that the closed and longer valve structure in other studies may have been responsible for the potential benefit [8].

The greater stent migration rate in the ARVMS group in the current study is worthy of attention. The most common cause of stent dysfunction in the included studies was stent migration, a major cause of recurrent biliary obstruction. It may occur as a result of increased bile outflow pressure on the antireflux valve, or because the attachment of the antireflux valve at the duodenal end may prevent full expansion. Notably, Hamada et al indicated that chemotherapy may play

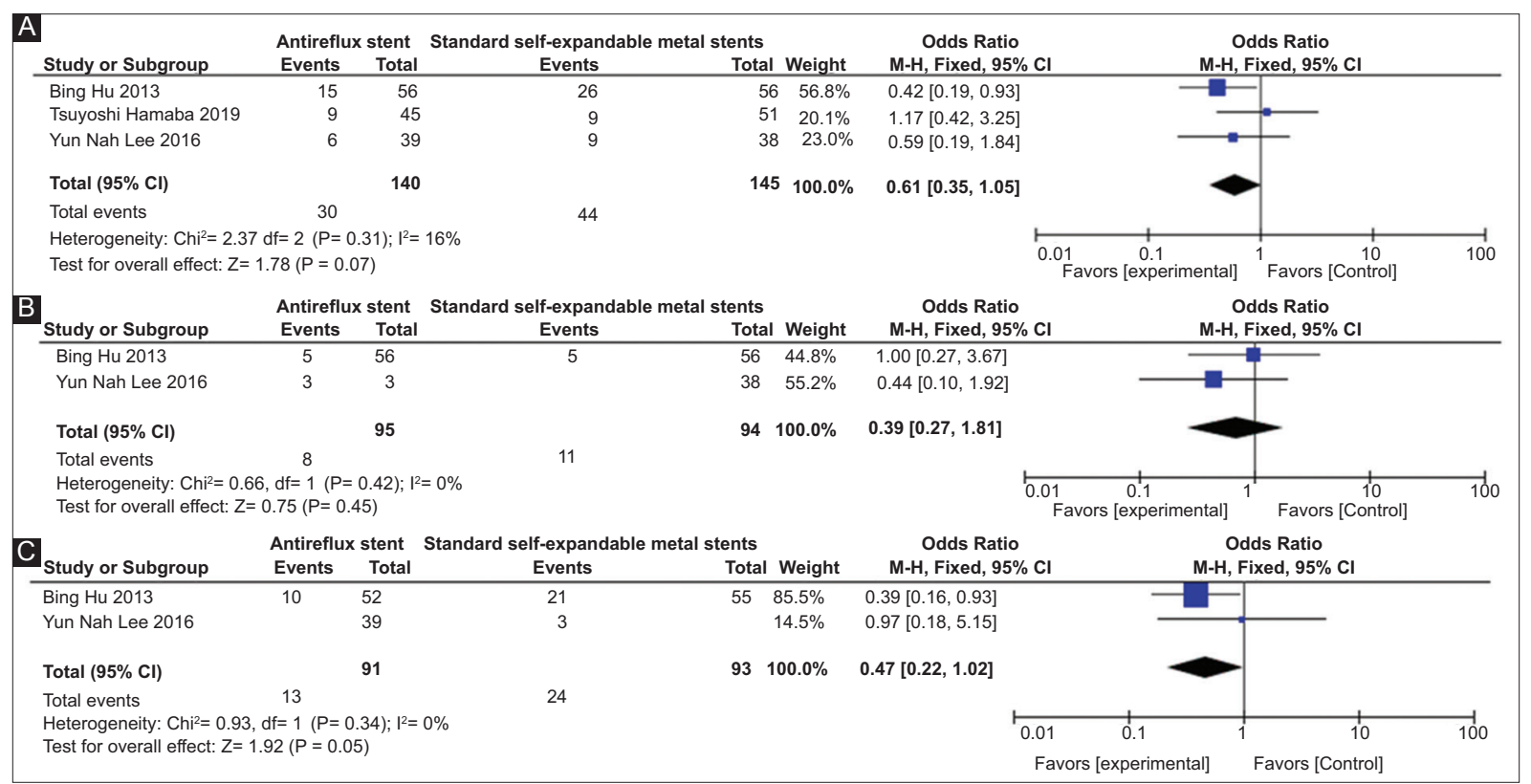

Figure 4 Forest plots representing odds ratios for (A) all adverse events, (B) early adverse events, and (C) late adverse events in the antireflux valve and the conventional self-expandable metal stent groups 


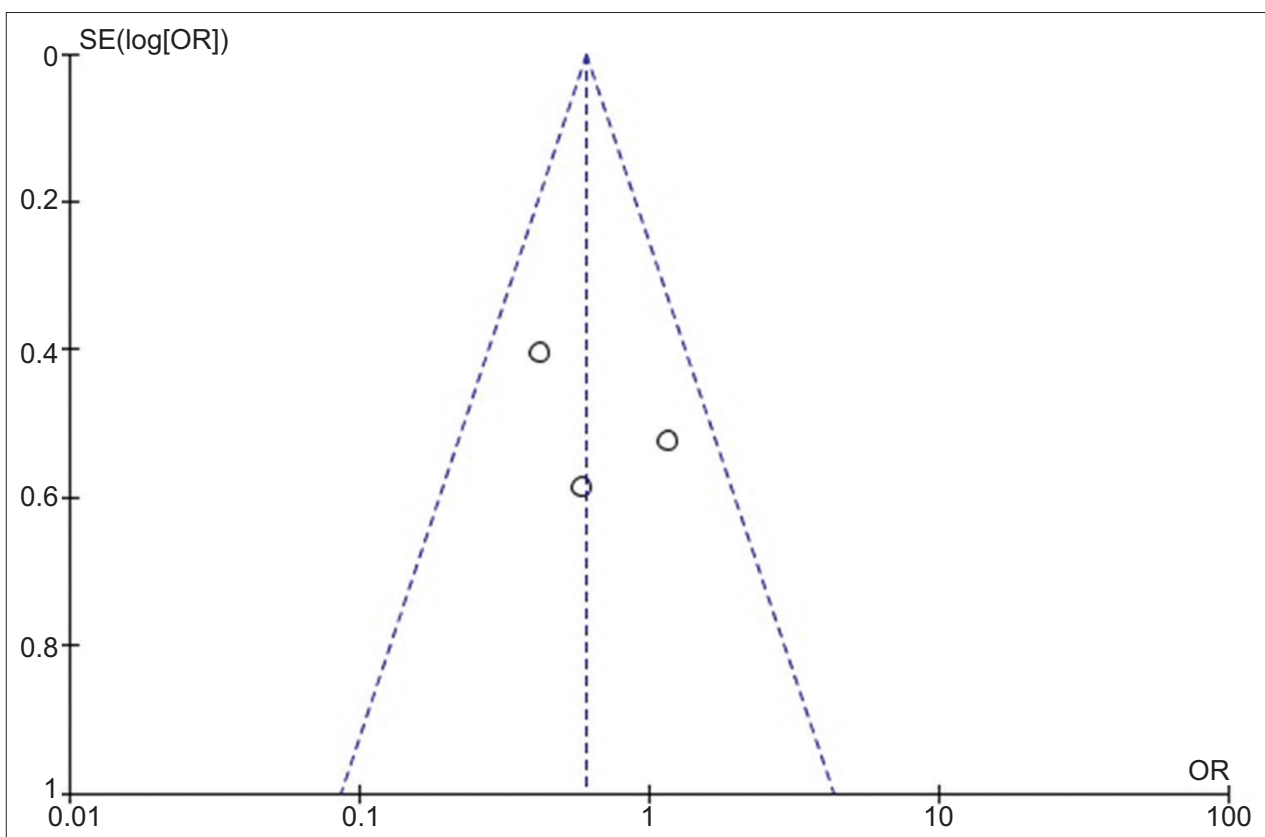

Figure 5 Funnel plot for the identification of publication bias for adverse events

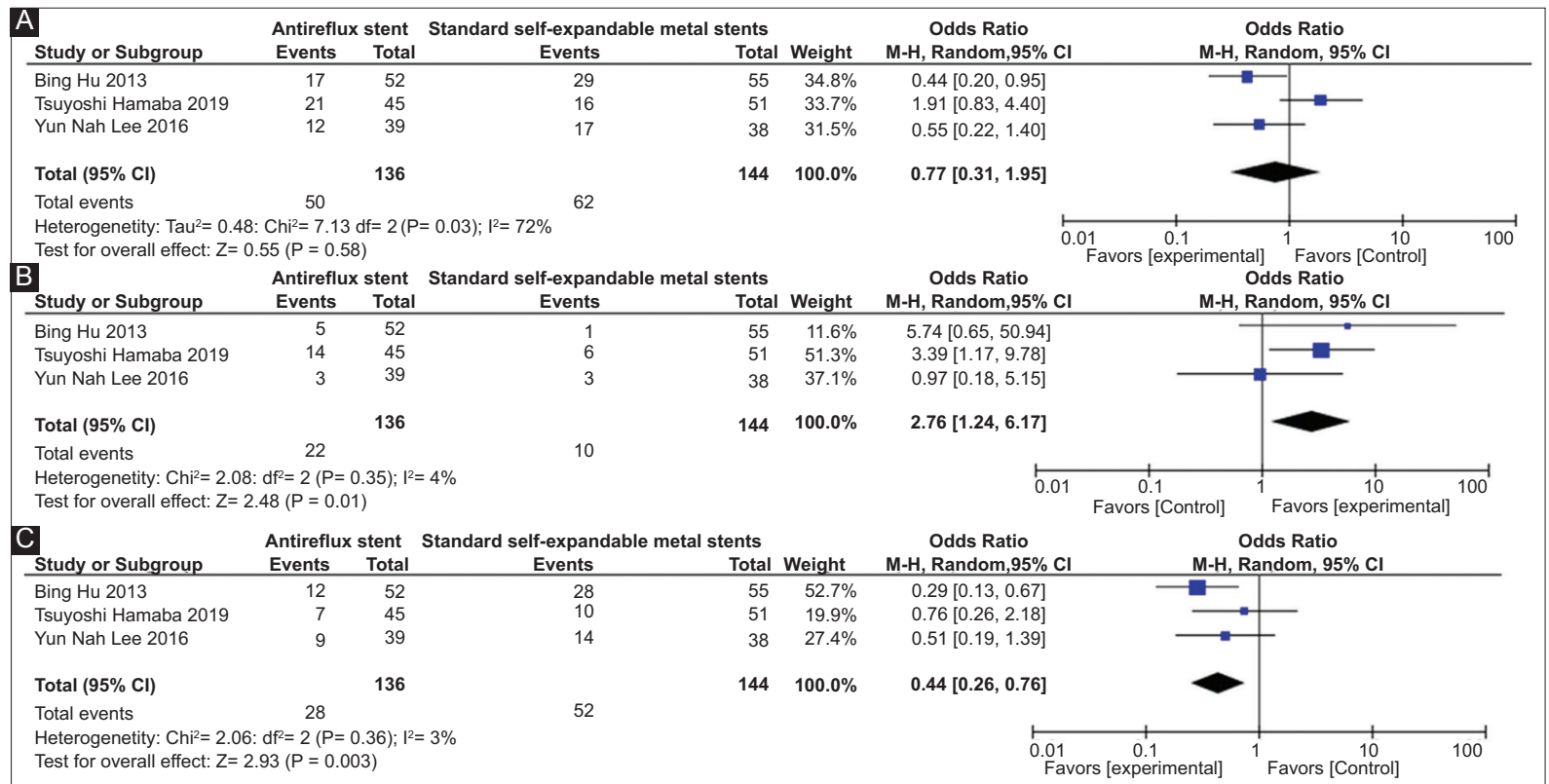

Figure 6 Forest plots representing odds ratios for (A) overall stent dysfunction, (B) stent migration, and (C) stent occlusion in the antireflux valve and the conventional self-expandable metal stent groups

a role in stent migration, as the between-group difference in stent migration was more pronounced in patients receiving chemotherapy $[8,18]$. Anti-migration properties, such as flared ends or low axial force, might overcome this drawback $[19,20]$.

Although not analyzed in the present study, $\mathrm{Hu}$ et al reported that, despite the better stent patency, ARVMS was not associated with a higher rate of patient survival. They iterated that the prognosis of these patients is more dependent on the tumor prognosis and therapy rather than the stent type [7].
However, their finding needs further confirmation in larger clinical trials to complete the picture on the ARVMS-SEMS comparison.

This study had some limitations. First, the low numbers of studies included and patients enrolled limit the generalizability and external validity of our findings. Furthermore, 2 of these were single-center studies. Second, owing to the nature of the procedure, patients, physicians, and outcome assessors were often not blinded to the treatment arms. Third, the limited 


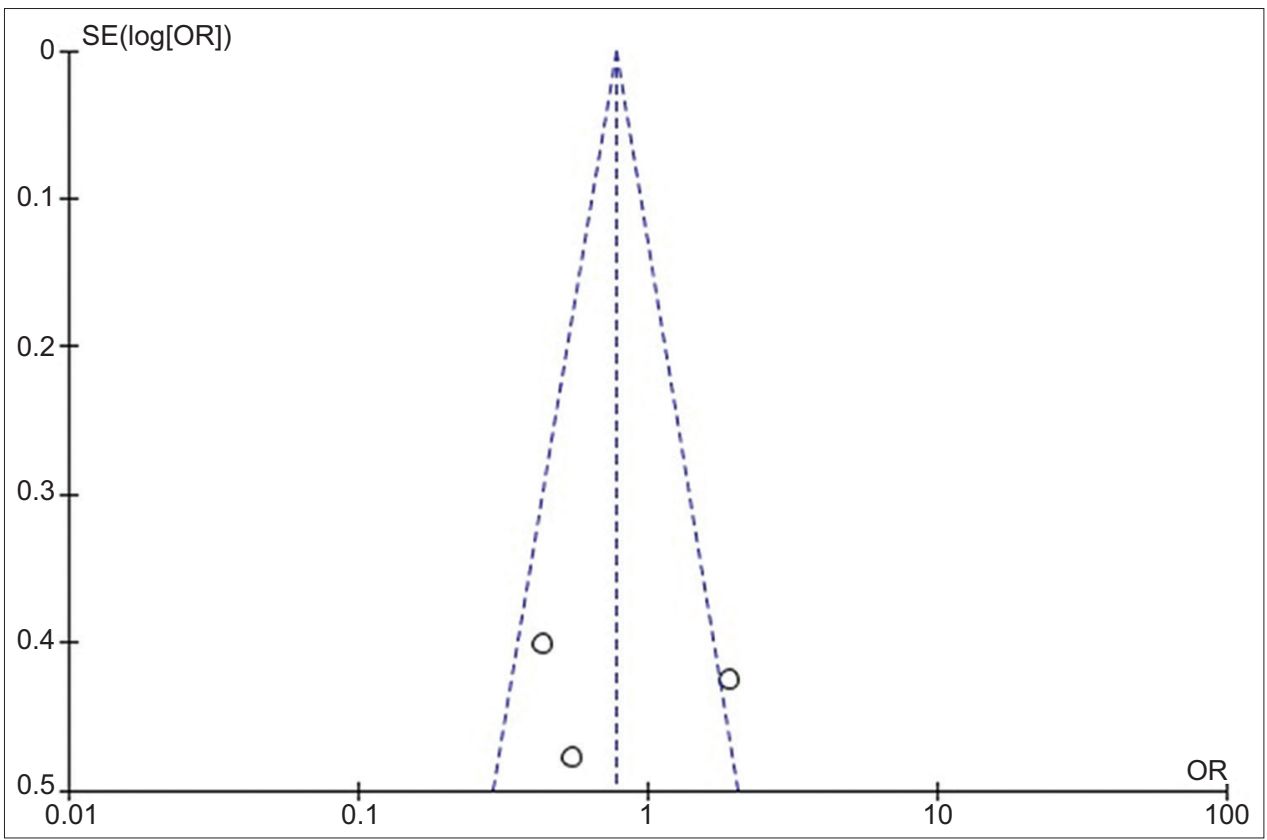

Figure 7 Funnel plot for the identification of publication bias for stent dysfunction

\section{Summary Box}

\section{What is already known:}

- The conventional self-expandable metal stent (SEMS) has been the gold standard for palliative treatment of non-resectable distal malignant biliary obstruction

- The antireflux valve metal stent (ARVMS) has been introduced as an alternative to SEMS to reduce the risk of cholangitis with SEMS

- Debate continues regarding the superiority of either of the 2 stent types

\section{What the new findings are:}

- No significant difference was found between ARVMS and SEMS regarding technical and clinical success rates

- Overall adverse events were comparable between ARVMS and SEMS; however, ARVMS had a lower risk of stent occlusion and a higher risk of migration

follow-up duration in all included studies precluded any long-term assessment of the durability of these stents. Fourth, different types of stents were used in the included studies: in the conventional SEMS arm, 2 studies [8,9] used covered SEMS, while the third study used uncovered SEMS [7]; in the ARVMS arm, 1 study used fully covered stents [8], while the other 2 used partially covered stents [7,9]. The difference in the type of stents could affect some reported outcomes, such as stent migration. The limited number of studies included in our meta-analysis made it impossible to perform a subanalysis based on the stent type to adjust for this difference. Further multicenter trials with longer follow-up periods are needed to establish the superiority of either stent type in patients with distal MBO.

In conclusion, our study showed no significant difference between ARVMS and SEMS regarding technical and clinical success rates. Overall adverse events were comparable between the 2 arms; however, ARVMS was associated with a lower risk of stent occlusion and a higher risk of migration. Larger RCTs are required to verify the benefit of ARVMS in distal MBO patients.

\section{References}

1. Kitano M, Yamashita Y, Tanaka K, et al. Covered self-expandable metal stents with an anti-migration system improve patency duration without increased complications compared with uncovered stents for distal biliary obstruction caused by pancreatic carcinoma: a randomized multicenter trial. Am J Gastroenterol 2013;108:1713-1722.

2. Dumonceau JM, Tringali A, Blero D, et al; European Society of Gastrointestinal Endoscopy. Biliary stenting: indications, choice of stents and results: European Society of Gastrointestinal Endoscopy (ESGE) clinical guideline. Endoscopy 2012;44:277-298.

3. Cheng JL, Bruno MJ, Bergman JJ, Rauws EA, Tytgat GN, Huibregtse K. Endoscopic palliation of patients with biliary obstruction caused by nonresectable hilar cholangiocarcinoma: efficacy of self-expandable metallic Wallstents. Gastrointest Endosc 2002;56:33-39. 
4. Wasan SM, Ross WA, Staerkel GA, Lee JH. Use of expandable metallic biliary stents in resectable pancreatic cancer. Am J Gastroenterol 2005;100:2056-2061.

5. Okamoto T, Fujioka S, Yanagisawa S, et al. Placement of a metallic stent across the main duodenal papilla may predispose to cholangitis. Gastrointest Endosc 2006;63:792-796.

6. Misra SP, Dwivedi M. Reflux of duodenal contents and cholangitis in patients undergoing self-expanding metal stent placement. Gastrointest Endosc 2009;70:317-321.

7. Hu B, Wang TT, Wu J, Shi ZM, Gao DJ, Pan YM. Antireflux stents to reduce the risk of cholangitis in patients with malignant biliary strictures: a randomized trial. Endoscopy 2014;46:120-126.

8. Hamada T, Isayama $\mathrm{H}$, Nakai $\mathrm{Y}$, et al. Antireflux covered metal stent for nonresectable distal malignant biliary obstruction: a multicenter randomized controlled trial. Dig Endosc 2019;31:566-574.

9. Lee YN, Moon JH, Choi HJ, et al. Effectiveness of a newly designed antireflux valve metal stent to reduce duodenobiliary reflux in patients with unresectable distal malignant biliary obstruction: a randomized, controlled pilot study (with videos). Gastrointest Endosc 2016;83:404-412.

10. Moher D, Liberati A, Tetzlaff J, Altman DG; PRISMA Group. Preferred reporting items for systematic reviews and meta-analyses: the PRISMA statement. Ann Intern Med 2009;151:264-269, W64.

11. Higgins JP, Altman DG, Gøtzsche PC, et al; Cochrane Statistical Methods Group. The Cochrane Collaboration's tool for assessing risk of bias in randomised trials. BMJ 2011;343:d-5928.

12. Higgins JP, Green S. Cochrane handbook for systematic reviews of interventions. 2008.

13. Isayama $\mathrm{H}$, Komatsu $\mathrm{Y}$, Tsujino $\mathrm{T}$, et al. A prospective randomised study of "covered" versus "uncovered" diamond stents for the management of distal malignant biliary obstruction. Gut 2004;53:729-734.

14. Krokidis M, Fanelli F, Orgera G, Bezzi M, Passariello R, Hatzidakis A. Percutaneous treatment of malignant jaundice due to extrahepatic cholangiocarcinoma: covered Viabil stent versus uncovered Wallstents. Cardiovasc Intervent Radiol 2010;33:97-106.

15. Saleem A, Leggett CL, Murad MH, Baron TH. Meta-analysis of randomized trials comparing the patency of covered and uncovered self-expandable metal stents for palliation of distal malignant bile duct obstruction. Gastrointest Endosc 2011;74:321-327.

16. Leung JW, Liu Y, Chan RC, et al. Early attachment of anaerobic bacteria may play an important role in biliary stent blockage. Gastrointest Endosc 2000;52:725-729.

17. Leung JW, Liu YL, Chan RC, Ling TK, Cheng AF. Effects of adherence factors and human bile on bacterial attachment and biliary stent blockage: an in vitro study. Gastrointest Endosc 2002;56:72-77.

18. Nakai $Y$, Isayama $H$, Mukai $T$, et al. Impact of anticancer treatment on recurrent obstruction in covered metallic stents for malignant biliary obstruction. J Gastroenterol 2013;48:1293-1299.

19. Isayama $H$, Mukai $T$, Itoi $T$, et al. Comparison of partially covered nitinol stents with partially covered stainless stents as a historical control in a multicenter study of distal malignant biliary obstruction: the WATCH study. Gastrointest Endosc 2012;76:84-92.

20. Bordaçahar B, Perdigao F, Leblanc S, et al. Clinical efficacy of antimigration features in fully covered metallic stents for anastomotic biliary strictures after liver transplantation: comparison of conventional and anti-migration stents. Gastrointest Endosc 2018;88:655-664. 